\title{
Restricting the means of suicide by charcoal burning
}

Paul S. F. Yip, C. K. Law, King-Wa Fu, Y. W. Law, Paul W. C. Wong and Ying Xu

\section{Summary}

We conducted an exploratory controlled trial to examine the efficacy of restricting access to charcoal in preventing suicides from carbon monoxide poisoning by charcoal burning in Hong Kong. All charcoal packs were removed from the open shelves of major retail outlets in the intervention region for 12 months; in the control region, charcoal packs were displayed as usual. The suicide rate from charcoal burning was reduced by a statistically significant margin in the intervention region $(P<0.05)$ but not in the control region. We observed no significant change in the suicide rate using other methods in either location.

\section{Declaration of interest}

None.
Charcoal burning has emerged as a common method of suicide in Hong Kong, rising from 2\% of all suicides in 1998 to over $26 \%$ in $2003{ }^{1-4}$ It has also spread rapidly to other Asian societies via the media and the internet (online Fig. DS1). ${ }^{5}$ In addition, it has been shown that the sociodemographic and clinical characteristics of people who die using this method are different from those who complete suicide by other means, and that the former are less likely to have a history of psychiatric illness or substance misuse. ${ }^{3,4}$ Thus, the traditional clinical approach for identifying proximate, individual-level risk factors might not be an effective way of preventing such suicides. ${ }^{6}$ Limiting the public's access to charcoal could be an effective means of preventing charcoal-burning suicides; it could also possibly prevent suicidal individuals from finding an alternative method of suicide. ${ }^{7-10}$ This exploratory study therefore sought to evaluate the efficacy of a communitybased intervention programme to prevent charcoal-burning suicides by limiting access to charcoal sales for a 12-month period.

\section{Method}

We selected two geographically adjacent districts with similar demographic and socioeconomic characteristics: Tuen Mun (the intervention region) and Yuen Long (the control region). In 2006 these two districts had 502000 and 534000 inhabitants respectively, accounting in total for $15.1 \%$ of the population of the Hong Kong Special Administrative Region of 6.9 million people (online Table DS1). Both districts were relatively socially and economically disadvantaged compared with other districts in the territory. In 2006 they also had higher rates both of suicide by charcoal burning (Tuen Mun 4.0 per 100 000, Yuen Long 5.2 per 100000 ) and all suicides (Tuen Mun 16.5 per 100 000, Yuen Long 14.2 per 100000$)$ than the entire territory (2.4 per 100000 for suicide by charcoal burning and 13.6 per 100000 for all suicides).

In the intervention district (Tuen Mun) access to charcoal was limited by removing all barbecue charcoal packs from the open shelves of major retail chains. Customers were required to ask a shop assistant for a pack, which the assistant would then retrieve from a locked container. This was designed to make access more difficult for people in a state of heightened distress.

The study period began on 1 July 2006 and continued for 12 months, ending on 30 June 2007. It was a double-blind trial. The programme was not publicly announced, and the general public and frontline staff members in the supermarket chains in Tuen Mun did not know about it. The frontline staff were briefed by the management to the effect that the removal of barbecue charcoal packs from open shelves was solely an administrative change. Therefore, any observed alterations in the number of charcoal-associated suicides were unlikely to have been related to attitude changes among people living in the intervention district or to behavioural alterations among staff of the participating chains. We conducted two on-site quality checks in August 2006 and May 2007 to ensure that every shop was compliant with the requirements. Waiting time to obtain charcoal varied depending on the volume of traffic in the stores, with an average of about $10 \mathrm{~min}$. The sales volume of charcoal packs from the chains was slightly affected, as reported by the participating stores, but no serious complaint was received from any customer in the intervention district. Information about completed suicides was made available from the coroner's court. To ensure the accuracy and quality of suicide data, we reviewed all related police investigation reports, medical files and suicide notes of every reported case of suicide recorded at the coroner's court. We used the dead person's residence rather than the site of the death scene to classify the location of the suicide death (over $80 \%$ of the charcoal-burning deaths occurred at the deceased's home). ${ }^{3}$ We performed two independent $t$-tests on the difference in suicide rates between Tuen Mun and Yuen Long for charcoal-burning suicides in the pre-intervention period to assess whether any significant changes in the intervention period could be attributed to a regression to the mean from different starting points, rather than to the net effect of the intervention. We further used a Poisson regression model to assess the effect of the intervention with respect to age and gender between the two districts (for details see online data supplement). To assess the method substitution effect, we separately tested the differences in changes in the suicide rate for other methods with respect to the changes in the suicide rate for charcoal-burning poisoning.

\section{Results}

Table 1 gives the number and rate of suicides in men and women combined, by method, for Tuen Mun, Yuen Long and all districts in Hong Kong for the periods July 2005 to June 2006 and July 2006 to June 2007, i.e. the preceding year and the year of the intervention (complete results by gender and method are shown in online Table DS2). During the intervention period we observed an overall $5.7 \%$ reduction in the suicide rate for all districts from the baseline level of 14.1 per 100000 to 13.3 per 100000 , with reductions of $11.5 \%$ (from 2.6 to 2.3 per 100000 ) and $5.2 \%$ (from 11.6 to 11.0 per 100000 ) for charcoal-burning and non-charcoalburning suicides respectively. The observed changes in the suicide rate before and after the intervention in Tuen Mun (from 17.9 to 


\begin{tabular}{|c|c|c|c|c|c|c|c|}
\hline & \multirow[b]{2}{*}{ Period $^{a}$} & \multicolumn{2}{|c|}{ Intervention Tuen Mun } & \multicolumn{2}{|c|}{ Control Yuen Long } & \multicolumn{2}{|c|}{ All districts } \\
\hline & & Cases, $n$ & Rate $^{b}$ & Cases, $n$ & Rate $^{b}$ & Cases, $n$ & Rate $^{b}$ \\
\hline \multirow[t]{2}{*}{ Charcoal burning } & Pre-intervention & 21 & $4.3^{*}$ & 16 & 3.0 & 176 & 2.6 \\
\hline & Intervention & 10 & 2.0 & 23 & 4.3 & 159 & 2.3 \\
\hline \multirow[t]{2}{*}{ Other methods } & Pre-intervention & 67 & 13.6 & 51 & 9.6 & 790 & 11.6 \\
\hline & Intervention & 50 & 10.2 & 43 & 8.1 & 758 & 11.0 \\
\hline \multirow[t]{2}{*}{ All methods } & Pre-intervention & 88 & $17.9^{*}$ & 67 & 12.7 & 966 & 14.1 \\
\hline & Intervention & 60 & 12.2 & 66 & 12.5 & 917 & 13.3 \\
\hline
\end{tabular}

12.2 per 100000 ) and Yuen Long (from 12.7 to 12.5 per 100000 ) were minus $31.8 \%$ and minus $1.6 \%$ respectively. We attribute this difference mainly to the reduction in suicides by charcoal burning in Tuen Mun by $53.5 \%$ (from 4.3 to 2.0 per 100000 ). At the same time we observed an increase of $43.3 \%$ in the suicide rate from charcoal-burning poisoning in Yuen Long (from 3.0 to 4.3 per $100000)$. Furthermore, the differences in the charcoal-burning suicide rate between Tuen Mun and Yuen Long in the pre-intervention period were 4.3 and 3.0 per 100000 respectively, which were not statistically significant $(P=0.266)$. The results of the Poisson regression model also confirmed that the intervention in Tuen Mun was an effective way to prevent suicide by charcoal burning (online Table DS3). Finally, the change did not appear to divert potential suicide attempts to other methods. This suggests that the programme resulted in some fundamental changes to the epidemiology of charcoal-burning suicide in the intervention region during the intervention period.

\section{Discussion}

Our results strongly suggest the efficacy of limiting access to retail charcoal in preventing suicide by charcoal burning. The fact that the overall suicide rate also fell suggests that the intervention did not prompt a switch to alternative methods of suicide. The whole idea behind restricting means of suicide is not to remove the means from the mass market completely, but rather to set up barriers to obtaining lethal quantities of the means for self-destruction. Charcoal is not an essential or daily household item in urbanised societies such as Hong Kong and some cities in Taiwan and Japan. Instead, it is used mostly for recreational activities such as outdoor barbecues in Hong Kong, or for the practice of religious rituals such as ancestor worship in Taiwan. The readiness of the whole community to accept relatively minor restrictions on the sale of charcoal packs, which might cause the inconvenience of a delay in purchasing charcoal, has yet to be tested on a territory-wide level. Also, there was a slight downward effect on the sales of charcoal in the big chain stores; whether there would be a continued willingness on the part of these stores to implement these changes in other districts in the community remains unclear. However, the exploratory study should be confirmed by a cluster randomised trial involving a number of areas in the future.

The results of this study should be interpreted in light of the following limitations. There was a lack of replication in experimental units, i.e. only one region per study group. Further studies may be needed in order to be able to generalise the results to other districts with different profiles. The study used only a relatively short period of 1 year of suicide data as the baseline, and another year for the post-assessment for the control and intervention regions, to avoid any potential contamination effect over the study period. Charcoal remained available for purchase in some small shops at the barbecue sites located in the intervention district, although this would have had a minimal effect on the means used by suicidal persons.

Paul S. F. Yip, PhD, Hong Kong Jockey Club Centre for Suicide Research and Prevention, and Department of Social Work and Social Administration, University of Hong Kong; C. K. Law, PhD, Hong Kong Jockey Club (HKJC) Centre for Suicide Research and Prevention, University of Hong Kong, and Hong Kong Institute of AsiaPacific Studies, Chinese University of Hong Kong; King-Wa Fu, PhD, HKJC Centre for Suicide Research and Prevention, University of Hong Kong and Journalism and Media Studies Centre, University of Hong Kong, China; Y. W. Law, MSW, Paul W. C. Wong DPsych, HKJC Centre for Suicide Research and Prevention, University of Hong Kong; Ying $\mathbf{X u}, \mathrm{PhD}$, Singapore Clinical Research Institute, Singapore

Correspondence: Paul Yip, HKJC Centre for Suicide Research and Prevention, University of Hong Kong, Hong Kong. Email: sfpyip@hku.hk

First received 22 Feb 2009, final revision 30 Feb 2009, accepted 7 Oct 2009

\section{Funding}

This research was supported by the Bureau of Labour and Welfare of the Hong Kong Government of Special Administrative Region.

\section{Acknowledgements}

We are grateful for the voluntary participation of the five retail chains China Resource Vanguard (4 shops), ParkNShop (12 shops), Wellcome (16 shops), 7-Eleven (5 shops) and Circle K (3 shops). We further express our gratitude to the South China Morning Post feature writer simon Parry for his support in suicide prevention work, and the many useful comments from the reviewers

\section{References}

1 Yip PSF. Suicide in Asia: Causes and Prevention. Hong Kong University Press, 2008

2 Chan KP, Lee DT, Yip PS. Media influence on suicide. Media's role is double edged. BMJ 2003; 326: 498.

3 Chan KP, Yip PS, Au J, Lee DT. Charcoal-burning suicide in post-transition Hong Kong. Br J Psychiatry 2005; 186: 67-73.

4 Yip PSF, Lee DTS. Charcoal-burning suicides and strategies for prevention. Crisis 2007; 28: 21-7.

5 Liu KY, Beautrais A, Caine E, Chan K, Chao A, Conwell Y, et al. Charcoa burning suicides in Hong Kong and urban Taiwan: an illustration of the impact of a novel suicide method on overall regional rates. J Epidemiol Community Health 2007; 61: 248-53.

6 Chen YY, Yip PSF. Rethinking suicide prevention in Asian countries. Lancet 2008; 372: 1629-30.

7 Mann JJ, Apter A, Bertolote J, Beautrais A, Currier D, Haas A, et al. Suicide prevention strategies: a systematic review. JAMA 2005; 294: 2064-74.

8 Beautrais AL. Effectiveness of barriers at suicide jumping sites: a case study. Aust N Z J Psychiatry 2001; 35: 557-62.

9 Law CK, Yip PS, Chan WS, Fu KW, Wong PW, Law YW. Evaluating the effectiveness of barrier installation for preventing railway suicides in Hong Kong. J Affect Disord 2009; 114: 254-62.

10 Hawton K, Townsend E, Deeks J, Appleby L, Gunnell D, Bennewith O, et al. Effects of legislation restricting pack sizes of paracetamol and salicylate on self-poisoning in the United Kingdom: before and after study. BMJ 2001; 322 1203-7. 